\title{
Wireless Multi-Hop Network Data Transmission of Multi-Parameter Joint Control
}

\author{
Liang Zong ${ }^{1}$, Yudan Ning ${ }^{2, *}$, Chenglin Zhao ${ }^{1}$, Gaofeng Luo ${ }^{1}$ and Baoxing Pu ${ }^{3}$ \\ ${ }^{I}$ College of Information Engineering, Shaoyang University, Shaoyang 422000, China \\ ${ }^{2}$ Department of Information Technology and Creativity, Shaoyang Polytechnic, Shaoyang 422000, China \\ ${ }^{3}$ School of Data Science and Software Engineering, Wuzhou University, Wuzhou, 543002, China
}

Received 1 February 2021; Accepted 21 April 2021

\begin{abstract}
The multi-hop routing composed of wireless nodes in wireless multi-hop network can cause serious data loss, which affects the transmission performance of multi-hop network. To reveal the influence of data loss on the transmission performance of wireless multi-hop networks, this study proposed a transmission control method based on multiparameter judgment of network status. A judgment model of wireless multi-hop network condition was established according to the feedback information of several parameters. The congestion status of wireless multi-hop network and the random errors in data transmission were analyzed on the basis of three parameters, namely, inter-packet delay difference, short-term throughput, and packet out-of-order delivery ratio. After evaluating the wireless multi-hop network status, the growth factor of the congestion window was introduced combined with the number of hops in the link and the weighted packet loss rate, and the window growth rate was analyzed. Moreover, the accuracy of the scheme was verified by experiments. Results demonstrate that the growth rate of the congestion window can improve the transmission rate of wireless multi-hop networks. Compared with the traditional Transmission Control Protocol (TCP) scheme, the proposed scheme improves the throughput by nearly $35 \%$ in the chain topology scenario of wireless multi-hop networks. The study provides reference for evaluating the performance of data transmission in wireless multi-hop networks.
\end{abstract}

Keywords: Multi-hop network, Congestion control, Random loss, Delay difference

\section{Introduction}

Considering that wireless multi-hop networks, such as Mobile Ad hoc NETwork (MANET), wireless mesh network (WMN), Ad hoc network, and even Vehicle Ad-hoc NETwork, do not need advance lay of infrastructure, they have the advantages of high flexibility, self-organized, and no centralized coordinator [1-4]. With the development of modern communication technology, wireless multi-hop networks have been widely used in many occasions, such as in post-disaster rescue and forest and marine environment detection in remote areas.

In wireless multi-hop network, the multi-hop routing is composed of multiple wireless nodes. Each node in the network exchange data uses wireless links. If two nodes can communicate directly, then data exchange can be realized. If nodes are not in the communication range, then they can communicate by means of multi-hop relay data forwarding by other nodes, which causes random data loss. Moreover, the nodes in the network are self-organized. In other words, they can realize autonomous connections in the topological structure. Once a path is unconnected, they will look for other connected paths. Nodes can be either static or dynamic in the network. If the nodes are dynamic, then the multi-hop network is a dynamic ad hoc network. If the nodes are static, then the multi-hop network is static. Nodes in the network can be taken as data senders, receivers, or transferers of routes. Finally, the network can realize communication without the assistance of infrastructure (e.g., base stations

*E-mail address: yudan_ning@163.com

ISSN: 1791-2377 @ 2021 School of Science, IHU. All rights reserved.

doi:10.25103/jestr.142.10 and Wi-Fi hotspots). Therefore, the nodes have distributed control. Data transmission in wireless multi-hop networks faces many challenges because of the above-mentioned characteristics. One of the main problems is the data loss. Topological connection in the network may be intermittent during data transmission, thereby increasing the difficulty of transmission control in the network [5-7].

Scholars have carried out numerous studies on transmission control in wireless multi-hop networks [8-10]. However, the multi-hop routing characteristics of wireless multi-hop network lead to the low efficiency of data transmission and throughput. Thus, changing the amount of data sent by the congestion window accurately according to different network states has become an urgent problem to be solved.

This study proposed a congestion control method based on the multi-parameter judgment of network state. The state of multi-hop network is judged according to different parameters, so that data loss can be estimated in time. The sending value of the congestion window is changed according to different data loss conditions, thereby providing reference for improving the performance evaluation of wireless multi-hop network.

\section{State of the art}

At present, scholars have carried out many studies on the transmission control of multi-hop networks. The early transmission control protocol (TCP) applicable to wired networks has the problem of serious performance 
degradation in wireless ad hoc networks. The reason is that TCP is constrained by many factors in wireless multi-hop networks, such as routing failure, wireless channel competition, exposed terminals, and hidden terminals.

Considering the serious fairness of $\mathrm{WMN}$ at the transport layer, Arianpoo [11] proposed a distributed network monitoring mechanism using cross-layer method, which deployed reinforcement learning technology to achieve fair resource allocation among nodes in WMN settings. A distributed cooperation mechanism, in which each node that hosted TCP source monitored the network and adjusted the TCP parameters dynamically according to the network state, was established. This scheme improved TCP fairness in wireless multi-hop networks. However, no specific scheme improves the throughput of transmission control.

Considering that data packet reordering and noncongestion loss in heterogeneous networks may be misinterpreted as congestion loss, Leung [12] developed an intelligent TCP sender model to distinguish congestion problems from non-congestion problems. More reliable packet loss and network congestion signals can be constructed on conventional channels with frequent errors. This scheme has remarkable resistance to packet reordering and non-congested packet loss. It only needs to modify the sender of the TCP, which was convenient for future deployment. However, the workload of two timers was increased in this scheme, thereby causing adverse impact on the data sender.

Jude [13] studied the impact of data loss on the throughput and fairness performance of wireless multi-hop networks. A congestion control method based on network assistance and window utilization was proposed. The scheme started the congestion control process on the basis of the queue level notification of relay nodes and reduced the sending rate according to the utilization level of TCP flow. The throughput, flow fairness, and end-to-end delay performance were improved, but the TCP-friendliness was neglected. Routing protocol was a key factor that influenced the performance of data transmission because nodes in multi-hop network play the role of data forwarding. Shenoy [14] simulated ad hoc on-demand distance vector routing (AODV), ad-hoc on-demand multipath distance Vector routing, and dynamic source routing (DSR) protocols to find an ad hoc network routing protocol that meets the requirements of ad hoc scenes. Experimental simulation showed that AODV protocol has better performance than other routing protocols. However, the study did not provide a new routing protocol.

Atsuta [15] studied the characteristics of mesh network based on IEEE 802.11s, measured the performance of TCP CUBIC and TCP-BBR on mesh network, and evaluated its performance. The study revealed that the probability of data loss decrease the performance of mesh network with the increase in delay, but specific TCP design scheme was not given. Considering that the multi-hop network of RFID readers uses TCP as the transport layer protocol, Radha [16] proposed a rate-based transmission algorithm, which was implemented as a layer between TCP and the network layer. The proposed algorithm can ensure that packets were sent successively with low delay. This scheme was superior to TCP New Reno algorithm in terms of throughput, end-toend delay, link layer contention, and routing failure performance evaluation.

Park [17] proposed a mechanism to enhance the performance of TCP in wireless low-power and lossy networks by improving the feedback information of TCP.
This scheme was only used in the intermediate process of border routers that intervene in TCP communication, and the function was achieved through the RTT of data flow. The operation of data packets or existing protocols will not be affected. However, this scheme did not consider TCP friendliness. Wallaschek [18] introduced a novel TCP scheme that could generate congestion control algorithm in a data-driven way automatically. The fairness between throughput and flow was improved to some extent. However, the data transmission protocol in multi-hop nodes, which was not conducive to end-to-end data transmission, was modified.

Molia [19] discussed the TCP possibility of wireless multi-hop networks in machine learning. The study pointed out that MANET was a resource-constrained network with limited power supply and processing resources. The computation of machine learning consumed a lot of resources, which will inevitably affect the application of machine learning in multi-hop networks. However, the scheme failed to provide a specific machine learning scheme in TCP. Huang [20] redesigned the TCP congestion control in the wireless network of mobile applications and proposed the QUIC-TCP congestion control algorithm. The algorithm used Lyapunov method to establish a TCP fluid model to achieve global convergence and stability. However, this method needs mass calculation data at the data sending end, and its application in resource-constrained multi-hop networks may be limited.

To realize a secure transmission of wireless ad hoc networks in eavesdropping environment, Hui [21] proposed a joint secure routing and power optimization algorithm for wireless multi-hop ad hoc networks, which could obtain the optimal transmission power between source nodes and relay nodes of each hop through a given path. The optimal routing between source and destination nodes can be obtained. This algorithm can protect the data transmission in eavesdropping environment, but it did not improve the transmission throughput of the network.

To solve the problem of intelligent data transmission of Internet of vehicle (IoV), Zhang [22] proposed an optimal routing method for data transmission based on Markov decision theory. The comparative analysis results of similar methods verified that the routing strategy had higher data transmission rate and shorter data transmission delay. The algorithm had certain reference significance in improving the data transmission of the IoV. However, the algorithm heavily relied on optimization analysis, and the data calculation consumes a great amount of resources, which was a challenge for wireless multi-hop networks.

With the emergence of delay-sensitive applications and ultra-low delay scenarios, Zuo [23] analyzed the delay sources according to the network hierarchical architecture and summarized the technologies for shortening delay. Three typical low-latency key scenarios and delay optimization techniques were proposed for different networks, and the possible opportunities and challenges in the development of low-latency networks were introduced. However, no specific scheme in the low-latency scenario is provided. According to the diversified requirements of network application QoS in IoV terminal equipment, Ding [24] proposed a new congestion control algorithm driven on demand based on data priority and throughput evaluation. The algorithm used data priority and throughput ratio factor to calculate the weight value, sent data through different weight values, and dynamically adjusted the congestion window by evaluating path status. The algorithm met the 
requirements for service quality of different applications, but did not consider the friendliness and fairness of TCP.

According to the application analysis of TCP Vegas in wireless multi-hop networks, Jiang [25] developed an improved transmission control algorithm based on grey prediction theory. The congestion avoidance phase was enhanced in three aspects. The lower-layer parameters were considered in the throughput model to improve the accuracy of theoretical throughput. In addition, the convergence analysis of grey prediction was proved by using Lyapunov's second method. Compared with Vegas, the proposed algorithm had higher throughput and lower delay. However, this algorithm was not compared with other algorithms in his study.

Guo [26] analyzed the performance of aggregation schemes in wireless linear multi-hop networks, and discussed the influence of user datagram protocol (UDP) payload size on frame aggregation performance. Aggregation mechanism can improve the performance of multi-hop system to a certain extent, but it caused higher system delay. In poor channel environment, the system performance was seriously weakened. However, this study did not provide a specific solution.

The aforementioned studies focused on the evaluation of a single parameter and performance analysis in multi-hop networks, while the multi-parameter special analysis of wireless networks, especially the correlation of wireless multi-hop networks, was scarce. This study uses a method of multi-parameter feedback information to establish a score increasing model of data transmission in multi-hop networks. The network state is evaluated according to the inter-packet delay difference (IDD), short-term throughput (STT), packet out-of-order delivery ratio (POR), and packet loss ratio (PLR). The study results demonstrate that the value added of congestion window is related not only to the previous congestion window, but also to the growth factor, which provides a basis for improving the congestion control ability of wireless multi-hop networks.

The remainder of this study is organized as follows. Section 3 describes the multi-parameter congestion control algorithm and constructs a four-parameter joint evaluation model of network state. Section 4 performs experimental simulation to compare the transmission performance of different schemes in chain topology scenario. Finally, Section 5 summarizes this study and draws the conclusions.

\section{Methodology}

In the congestion control method of wireless multi-hop network, the data sender obtains the network state by evaluating many parameters, such as the round-trip time of data, the notification window of data receiver, the number of redundant acknowledge character and the returned data sequence number, so that whether the data loss is random packet loss or congestion packet loss can be judged. Multiparameter congestion control algorithm [27] not only solves the problem of congestion control in wireless multi-hop networks but also enhances its friendliness.

\subsection{Multi-parameter feedback}

In wireless multi-hop ad hoc networks, multi-parameter feedback is of great significance to judge true congestion and non-congestion correctly. Accurate network state judgment can improve TCP transmission performance in wireless multi-hop networks greatly. Correct judgment of the following network states in wireless multi-hop networks helps improve the TCP performance of the network.

Congestion state: When the network is congested, the transmission of wireless multi-hop network should adopt the same congestion control behavior as traditional TCP to reduce the data transmission rate. Congestion indicates the transmitted data overflows because of the cache of certain or some nodes.

Channel error state: When the data are in channel error state, the transmitted data are randomly lost. The sending end of the wireless multi-hop network can only retransmit the lost data without slowing down the data transmission.

Routing change state: When data are transmitted between the sender and the receiver, the transmission path may change due to the movement of nodes and the change in surrounding environment. The receiver in the wireless multihop network may experience a short period of disordered data arrival or data loss. The sender should re-estimate the bandwidth along the new path, set the current sending window as the current slow start threshold, and start the congestion avoidance phase.

Connection interruption state: If the data transmission path is interrupted for too long, then it will lead to timeout retransmission. However, if the interruption time is short, then the sender should freeze the congestion window and the current state. At this time, the sending end checks the network condition and reestablishes the connection periodically. Once the connection is established, it will execute the route change state.

In the four above-mentioned states, the loss of data packets may result from many factors. For example, the route change may make the data flow to a certain aggregation node, resulting in network congestion. Similarly, channel errors can also lead to transmission errors in the data link layer of the network, thereby causing route change or link interruption. Therefore, the congestion state has the highest priority in the network states. The three other states are only considered in the non-congested state.

In wireless networks, especially multi-hop networks, the network state can hardly be determined according to a single parameter, and it is necessary to combine with other related parameters. For the transport layer, if cross-layer feedback information is not used, then few parameters will be obtained. According to the limited information, the multiparameter congestion control algorithm proposes four parameters to estimate the IDD, STT, (POR, and PLR of continuous data packets and determine the network state.

The IDD is defined as

$I D D=\left(A^{i+1}-S^{i+1}\right)-\left(A^{i}-S^{i}\right)$

where $S^{i}$ is the time stamp when the data sender sends the i-th data packet, and $A^{i}$ is the time stamp when the i-th data packet is received by the data receiver. The delay difference of continuous data packets reflects the congestion degree of the network through the fluctuation of the time before and after the data packets in one-way transmission, but it has minimal influence on random packet loss.

STT is defined as

$$
S T T=\frac{N_{p}(T)}{T}
$$


where $N_{p}(T)$ is the number of data packets received in unit time period $T$. Similar to the IDD, STT can also evaluate the congestion state of the network. The former evaluates the congestion within a unit time interval, whereas the latter evaluates the congestion within a period of time. It is suitable to evaluate congestion combined with the two parameters. For non-congested parameters, the following definitions are presented.

POR is defined as

$$
P O R=\frac{N_{0}(T)}{P_{n}-P_{n-1}} \times 100 \%
$$

where $N_{0}(T)$ is the number of out-of-order packets received by the data receiver in a unit time period T, and $P_{n}$ is the maximum serial number of out-of-order packets received by the data receiver in the n-th unit time period $T$. As analyzed, a large amount of data will be out of order at the data receiver because of the change or interruption of the path in the transmission of wireless multi-hop networks. Therefore, this parameter can reflect whether the path changes or is interrupted in data connection indirectly. The ratio of out-of-order packets also has the following definition.

Packet loss rate (PLR) is defined as

$$
P L R=\left|1-\frac{N_{0}(T)}{P_{n}-P_{n-1}}\right| \times 100 \%
$$

where $P_{n}$ is the maximum serial number of out-of-order packets received by the data receiver in the $\mathrm{n}$-th unit time period T, and $N_{p}(T)$ is the number of data packets received in the unit time period $T$. This parameter evaluates the data loss in the unit time period of data reception, thereby reflecting the probability of data packet error caused by bit error rate in wireless communication link.

\subsection{Network state judgment}

In the network state of multi-parameter evaluation, if the IDD fluctuates greatly and the STT is low, then the data transmission in the network is likely to be congested. Otherwise, no congestion may occur in the network, and other network states will be further evaluated. When the POR fluctuates greatly, a large number of data packets will be out of order because of the changes in routing during transmission. When the PLR fluctuates greatly, data loss is caused by bit error rate of wireless link. In addition, link interruption can be judged according to STT. If the path of data transmission is interrupted, then the STT will approach zero in a certain time interval.

To be specific, congestion identification and feedback are carried out by using IDD, STT, POR, and PLR of continuous data packets. Whether data loss is congested is judged by combining the two parameters of delay difference and STT of continuous data packets. When IDD is high $(\mathrm{H})$ and STT is low (L), the network is congested. Otherwise, the network is not congested. Appropriate threshold values of high and low values should be set for IDD and STT. The network is congested when IDD is higher than the high value and STT is lower than the low value. In other cases, the network is considered not congested. Setting high and low values, which are determined by the specific multi-hop network environment, is important. The experiment in [27] shows that $30 \%$ of the two values can achieve good recognition results. In the data loss identification under the non-congested condition, the POR can also be introduced to judge whether random errors occur in data loss. The following scheme can be adopted. When the network is not congested, routing change, path interruption, and channel errors caused by node movement in wireless multi-hop networks can also lead to packet loss, and the sender will receive multiple repeated acknowledgments or time-out messages. If congestion control algorithm is used, then the performance of data transmission can be seriously affected. The reason for packet loss can be judged according to PLR and packet error sequence rate POR. If the network is not congested when the POR is high and exceeds a certain threshold value, then the route change causes the packet out of order. When PLR is high and exceeds a certain threshold, the channel error causes random packet loss. When STT is close to 0 , the path is interrupted (Fig. 1).

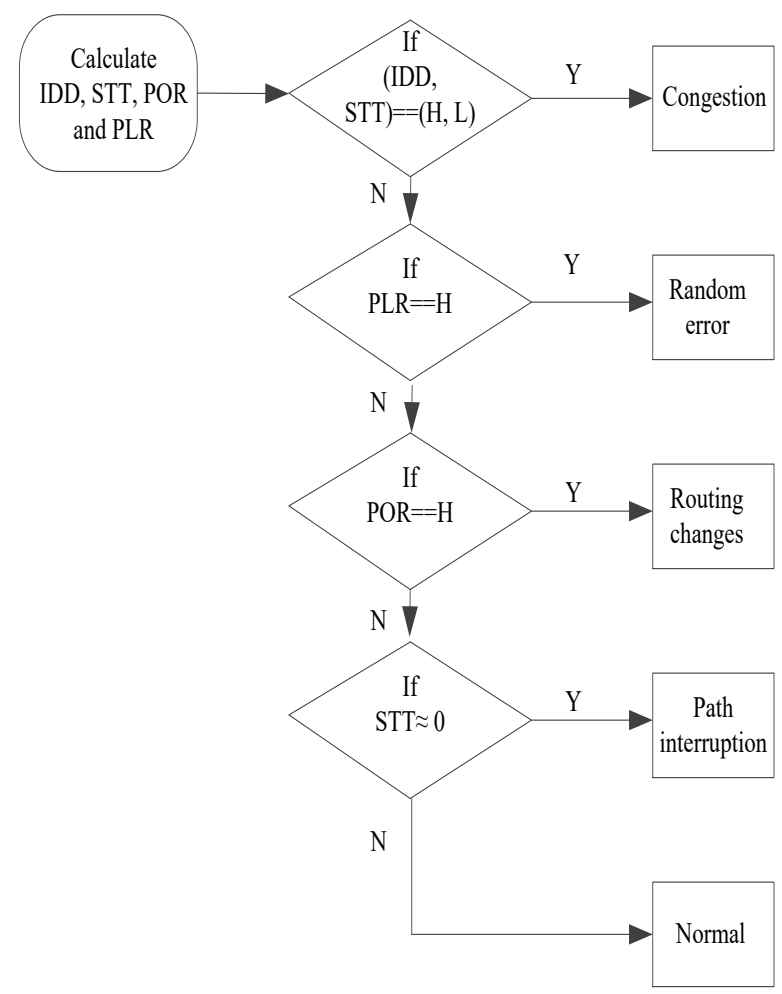

Fig. 1. Network state of multi-parameter feedback

\subsection{Congestion window setting}

Based on the evaluation of various network conditions by multi-parameter feedback, this study adopts a smaller window value for the congestion window setting of wireless multi-hop networks, and the proposed scheme uses a score increase window to control the window increase rate:

$$
W_{i+1}=W_{i}+\frac{\alpha}{W_{i}}
$$

where $\alpha$ is the growth factor of the congestion window, and the value interval is $0<\alpha \leq 1$. In addition to the previous congestion window, the increased value of the congestion window is also related to the growth factor $\alpha$, which is the value of adjusting the congestion window. In this scheme, the congestion window value of multi-hop network is determined according to the bandwidth delay product of multi-hop network. Specifically, the minimum value of the reasonable congestion window value is set as: 
$W_{i}=\frac{1}{8} \times B D P_{\text {upper limit }}$

where the bandwidth delay product upper limit of wireless multi-hop network is:

$B D P_{\text {upper limit }} \leq(n \times D+m \times A)$

where D and A are the sizes of forward path transmission data and reverse path transmission confirmation data, respectively. The $n$ and $m$ are the forward path hops and reverse path hops in the multi-hop network, respectively. The minimum value of Formula (6) cannot be less than 1, and the congestion window is increased according to Formula (5). The upper limit of the congestion window is:

$W_{\text {upper limit }}=\frac{1}{4} \times B D P_{\text {upper limit }}$

Limiting the growth interval of congestion window is beneficial to reduce the competition of wireless links caused by excessive data sent to the network. The growth factor in the congestion window is related to the PLR. The larger PLR indicates the smaller congestion window. The more accurate the evaluation of PLR indicates the more accurate value of the congestion window.

The value of $\alpha$ can be determined according to hop count and weighted PLR in the link. Specifically, when the link hop count is less than 7 hops and the PLR is low, the value of $\alpha$ is 0.01 . If the PLR is high, the value of $\alpha$ is 0.008 . When the link hop count is greater than 7 and the PLR is low, the value of $\alpha$ is 0.1 ; otherwise, the value of $\alpha$ is 0.08 .

The improved congestion algorithm mainly improves the transmission control of multi-hop networks from two aspects: adjusting the upper limit of congestion window and decreasing the increase rate of congestion window. When congestion is detected, the congestion window value will be adjusted to 1 ; otherwise, the congestion window value is calculated according to the above scheme, and its growth rate is adjusted according to Formula (5).

\section{Result Analysis and Discussion}

The experiment is simulated in OPNET, and the parameters are set as follows: IEEE 802.11 standard based on competitive scheduling scheme is selected for MAC layer, the data are sent continuously, the bandwidth is $2 \mathrm{Mbps}$, and the working frequency is $2.4 \mathrm{GHz}$. In the experiment, the transmission performance is simulated for TCP Reno (the traditional TCP scheme), Veno, and the proposed scheme. The experiment mainly tests the performance analysis of chain topology.

Fig. 2 shows a chain topology diagram in the wireless multi-hop network. A total of 12 nodes have a distance in the transmission area. In the experiment, the throughput of data links under different hop counts was tested. As the simplest chain topology, it is a static multi-hop network. The nodes only forward data, and connection reconstruction is not needed in mobile condition.

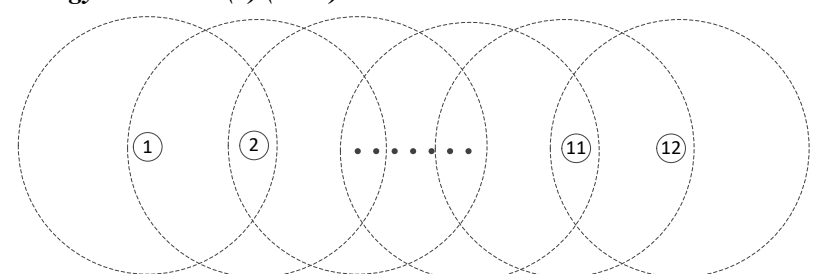

Fig. 2. Chain topology

Fig. 3 and Fig. 4 show the change in the data transmission rate in the wireless multi-hop network when the hop count is 6 . The test time is $60 \mathrm{~s}$, and the comparison scheme is the traditional TCP Reno and the proposed algorithm. The data transmission rate in the figure is the sum of the data transmission rates of the forward and backward paths. According to the interaction principle of the multi-hop network access layer, not only normal data transmission but also the transmission of interactive commands and acknowledgement messages between two adjacent nodes occur. Considering that the small amount of data of interactive commands, the throughput is mainly expressed by the data transmission rate and the statistics can reflect the bandwidth acquisition rate. The following diagram shows that the data transmission rate of the proposed algorithm is higher than that of the traditional TCP Reno. The restriction of the congestion window in the proposed scheme is beneficial to the data transmission of multi-hop networks, and the channel competition in the link layer is alleviated. The data transmission rate is maintained at approximately $205 \mathrm{kbps}$, and the fluctuation range is small. In Fig. 3, the data transmission rate of the proposed scheme is between $195 \mathrm{kbps}$ and $220 \mathrm{kbps}$, and the fluctuation range is $25 \mathrm{kbps}$. The delay difference and STT of continuous data packets can feedback the congestion judgment of multi-hop network to the increase rate of the congestion window. In this way, whether congestion occurs in multi-hop network has obvious influence on improving the data transmission rate of multihop network. Especially when no congestion is observed in the network, whether the data loss in multi-hop networks is caused by random channel errors can be further judged by introducing the PLR. The traditional TCP Reno exponentially increases the congestion window in the slow start phase, which obviously intensifies the competition of network data flow and is not conducive to the improvement of data transmission rate. The data transmission rate is maintained at approximately $143 \mathrm{kbps}$ with a wide fluctuation range. As shown in Fig. 4, the data transmission rate of TCP Reno is between $80 \mathrm{kbps}$ and $180 \mathrm{kbps}$, and the fluctuation range is $100 \mathrm{kbps}$, which is caused by the window of exponential increase in slow start stage. Also, once data loss occurs, TCP Reno judges that congestion occurs in multi-hop network. To avoid further data backlog, the congestion window is directly set to 1 , and the slow start phase is entered. This circular mode intensifies the fluctuation of multi-hop networks and decreases the data transmission rate substantially.

The relationship between the hop count and throughput in the chain topology of wireless multi-hop network is shown in Fig. 5. The average throughput of wireless multihop network decreases as the hop count in the forward path increases. Particularly, it decreases substantially when the hop count is between 2 and 4 . The throughput of TCP Reno decreases from $5.41 \times 10^{2} \mathrm{kbps}$ to $1.73 \times 10^{2} \mathrm{kbps}$, whereas the throughput of the proposed algorithm also decreases from $6.26 \times 10^{2} \mathrm{kbps}$ to $2.38 \times 10^{2} \mathrm{kbps}$. The throughput of the Veno scheme also declines to some extent. 
The main reason is that the transmission range and interference range covered by each node will obviously overlap because more nodes are found in the wireless multihop network. Therefore, for the wireless transmission of the monitoring channel in carrier sense mode, the probability of interference conflict of carriers in the wireless channel is relatively high, which is extremely unfavorable for data transmission. Therefore, the overall decline trend of the network throughput after 4 hops is relatively stable. The reasonable adjustment of the congestion window by the improved algorithm is conducive to slowing down the interference conflict of data in the wireless channel in carrier sense. According to Fig. 5, except for the high throughput at 2 hops, the throughput of the proposed scheme is mainly maintained from $2.38 \times 10^{2} \mathrm{kbps}$ to $1.34 \times 10^{2} \mathrm{kbps}$ at 4 hops to 12 hops, and the fluctuation range is $104 \mathrm{kbps}$. Similarly, the judgment of multi-hop network congestion by two parameters of delay difference and STT of continuous data packets can be fed back to the adjustment of congestion window to judge whether the multi-hop network is congested or not. As the hop count increases, the PLR can be introduced to further judge whether the data loss of the multi-hop network is caused by random channel errors in the non-congested environment. This step is helpful to improve the throughput of multi-hop network in the chain structure. The throughput of the improved algorithm is improved.

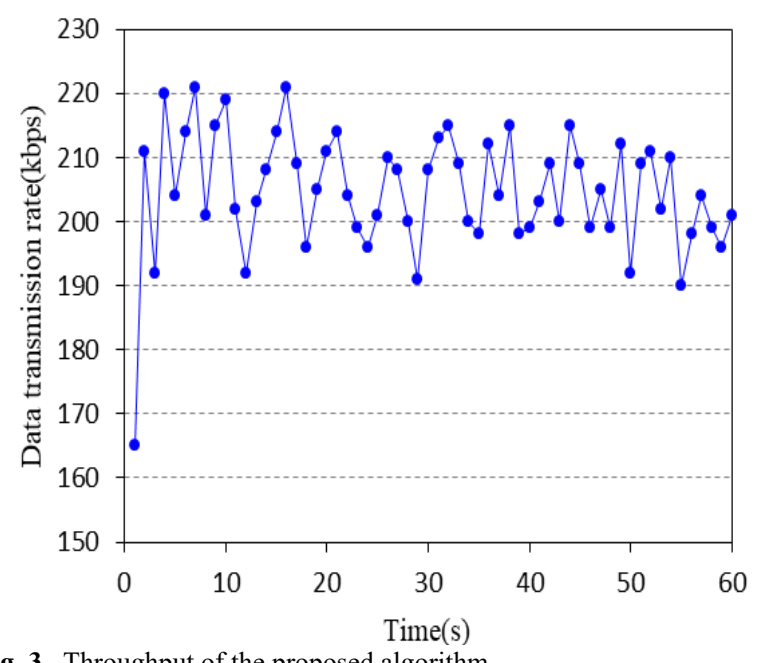

Fig. 3. Throughput of the proposed algorithm

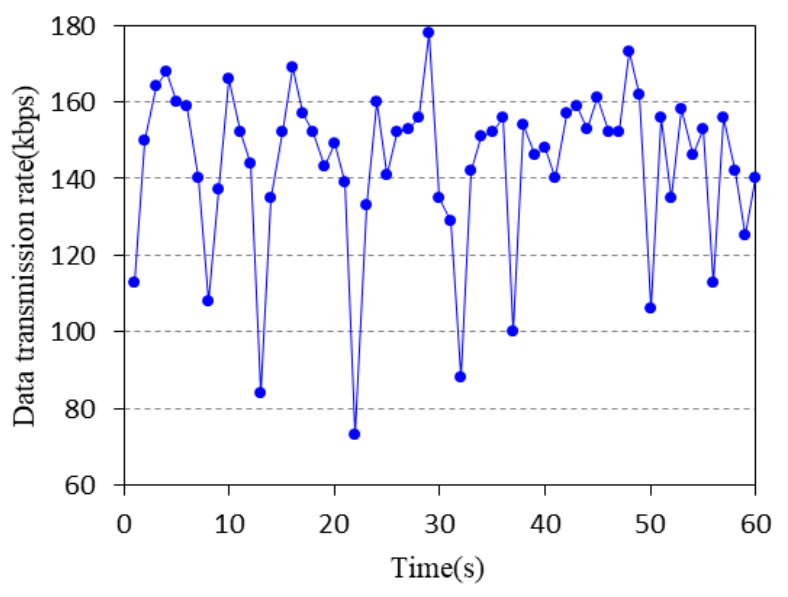

Fig. 4. Throughput of TCP Reno

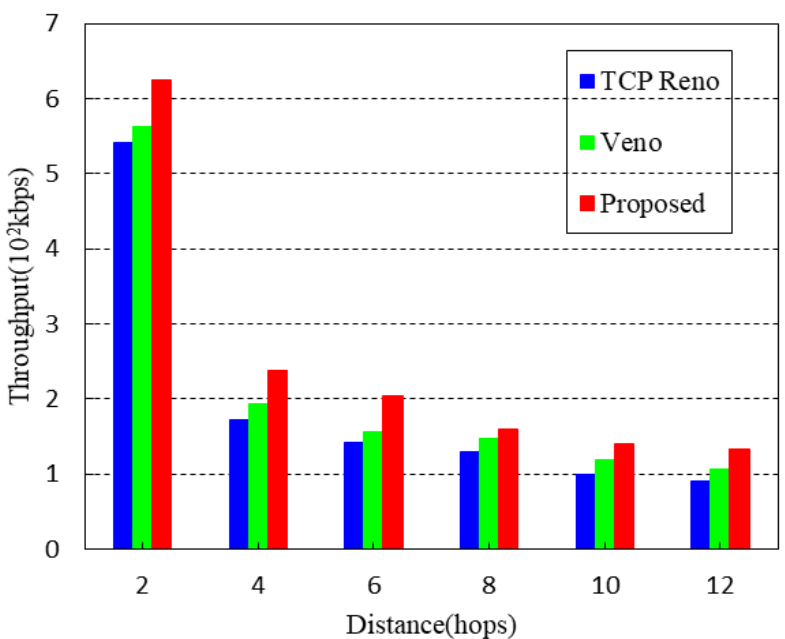

Fig. 5. Throughput of three transmission schemes

Compared with another two schemes, the throughput of the proposed scheme in chain topology has been improved to some extent. Table 1 shows the increase rates of the throughput of the proposed scheme compared with TCP Reno and Veno under different hop counts. With the increase in the hop count, the throughput of the proposed scheme is greatly improved compared with those of another two schemes, especially TCP Reno scheme. In general, with the increase in hop count, the increase rate of the throughput will become more obvious. As the hop count in chain topology increases, the probability of random loss in multihop network is greater, and the proposed scheme can distinguish whether congestion loss or random loss because of the timely feedback of multiple parameters, so that the status of multi-hop network can be reasonably judged and the congestion window size can be adjusted accurately. The throughput of multi-hop network in chain topology is significantly improved.

Table 1. Percentage of throughput improvement of the proposed scheme in the chain topology

\begin{tabular}{l|l|l|l|l|l}
$r$ hops & & & & & \\
schemes & 2 & 4 & 6 & 8 & 10 \\
\hline TCP Reno & $15.71 \%$ & $37.57 \%$ & $43.36 \%$ & $23.08 \%$ & $40.00 \%$ \\
\hline Veno & $11.33 \%$ & $23.44 \%$ & $31.33 \%$ & $8.04 \%$ & $17.15 \%$ \\
\hline
\end{tabular}

\section{Conclusions}

To explore the characteristics of congestion control in wireless multi-hop networks and reveal the relationship between data transmission and network status, this study investigated the characteristics of congestion window in wireless multi-hop network transmission control combined with multi-parameter joint feedback information and experimental study by analyzing four parameters and evaluating the network status. Finally, the following conclusions could be drawn:

(1) The IDD and STT are important criteria for judging network congestion.

(2) The POR and PLR are less sensitive to network congestion.

(3) The increase value of congestion window of data transmission in wireless multi-hop network is not only related to the previous congestion window but also to the 
growth factor $\alpha$. It uses a fractional increase window to control the rate of increase of the window.

By combining the congestion control experiment of wireless multi-hop network and theoretical study, a new scheme of multi-parameter joint feedback information was proposed to judge the network status. The established fractional increase window can reflect the network condition, which has a certain reference for the follow-up study of congestion control of multi-hop networks. Due to the lack of actual test equipment, future study will combine and modify the fractional increase window and the proposed model, so that the actual testing model can be more accurate.

\section{Acknowledgements}

This work was supported by the Hunan Provincial Natural Science Foundation (Grant No. 2020JJ4557), the Scientific Research Fund of Hunan Provincial Education Department (Grant Nos. 20B519, 19B512, and 19A446) and the Natural Science Foundation Project of Guangxi (No. 2020GXNSFAA238013).

This is an Open Access article distributed under the terms of the Creative Commons Attribution License.

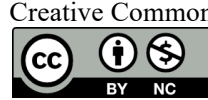

\section{References}

1. Conti M, Giordano S. "Mobile ad hoc networking: milestones, challenges, and new research directions". IEEE Communications Magazine, 52(1), 2014, pp. 85-96.

2. Sánchez-García J, García-Campos J M, Arzamendia M, Reina, D. G., Toral, S. L.,Gregor, D. "A survey on unmanned aerial and aquatic vehicle multi-hop networks: Wireless communications, evaluation tools and applications". Computer Communications, 119, 2018, pp. 43-65.

3. Redhu S, Anupam M, Hegde R M. "Optimal relay node selection for robust data forwarding over time-varying IoT networks". IEEE Transactions on Vehicular Technology, 68(9), 2019, pp. 9178-9190.

4. Gouissem A, Samara L, Hamila R, Al-Dhahir, N., Foufou, S "Multiple relay selection and beamforming in dual-hop amplifyand-forward relay networks". IEEE Systems Journal, 13(2), 2018 pp. $1534-1545$.

5. Lescisin M, Mahmoud Q H. "Ad-hoc messaging infrastructure for peer-to-peer communication". Peer-to-Peer Networking and Applications, 12(1), 2019, pp. 60-73.

6. Yi Y, Shakkottai S. "Hop-by-hop congestion control over a wireless multi-hop network". IEEE/ACM Transactions On Networking, 15(1), 2007, pp. 133-144.

7. Tan K, Jiang F, Zhang Q, Shen, X. "Congestion control in multihop wireless networks". IEEE Transactions on Vehicular Technology, 56(2), 2007, pp. 863-873.

8. Lochert C, Scheuermann B, Mauve M. "A survey on congestion control for mobile ad hoc networks". Wireless communications and mobile computing, 7(5), 2007, pp. 655-676.

9. Pióro M, Tomaszewski A, Capone A. "Maximization of multicast periodic traffic throughput in multi-hop wireless networks with broadcast transmissions". Ad Hoc Networks, 77, 2018, pp. 119-142.

10. Khaliq K A, Qayyum A, Pannek J. "Performance analysis of proposed congestion avoiding protocol for IEEE $802.11 \mathrm{~s}$ ". International Journal of Advanced Computer Science and Applications, 8(2), 2017, pp. 356-369.

11. Arianpoo N, Leung V C M. "How network monitoring and reinforcement learning can improve tcp fairness in wireless multihop networks". EURASIP Journal on Wireless Communications and Networking, 2016(1), 2016, pp. 278-293.

12. Leung K C, Lai C, Ding H. "TCP-NCL: A serialized-timer approach for enhancing TCP over heterogeneous wired/wireless networks". Computer Communications, 144, 2019, pp. 198-211.

13. Jude M, Auxilius J, Diniesh V C, Shivaranjani, M. "Throughput stability and flow fairness enhancement of TCP traffic in multi-hop wireless networks". Wireless Networks, 26, 2020, pp. 4689-4704.

14. Shenoy S U, Kumari S, Shenoy U K K. "Comparative Analysis of TCP Variants for Video Transmission Over Multi-hop Mobile Ad Hoc Networks". In: Proceedings of the International Conference on Computer Networks and Communication Technologies. Springer, Singapore: Springer, 2019, pp. 371-381.
15. Atsuta K, Kouya S, Sakamoto N. "Performance of TCP CUBIC and TCP BBR on IEEE802. 11s Mesh Network". Journal of Communications, 15(8), 2020, pp. 639-645

16. Radha R, Tyagi A K, Kathiravan K. "A Rate Control Algorithm to Improve TCP over RFID Reader Network". Singapore: Springer Press, Singapore, 2020, pp.13-24.

17. Park M, Paek J. "TAiM: TCP assistant-in-the-middle for multihop low-power and lossy networks in IoT". Journal of Communications and Networks, 21(2), 2019, pp. 192-199.

18. Wallaschek K L, Klose R, Almon L, Hollick M. "NEAT-TCP: Generation of TCP Congestion Control through Neuroevolution of Augmenting Topologies". In: Proceedings of the 2020 IEEE International Conference on Communications Workshops (ICC Workshops). Dublin, Ireland: IEEE, 2020, pp.1-6.

19. Molia H K, Kothari A D. "Appropriateness of Machine Learning Techniques for TCP with MANETs". In: Proceedings of 2019 the International Conference on Communication and Intelligent Systems (ICCIS 2019), Jaipur, India: Springer, 2019, pp. 503-511.

20. Huang H, Sun Z, Wang X. "End-to-End TCP Congestion Control for Mobile Applications". IEEE Access, 8, 2020, pp. 171628171642.

21. Hui H, Zhang X. "Joint Secure Routing and Power Optimization Algorithm for Wireless Ad Hoc Networks". Journal of Electronics and Information Technology, 42(12), 2020, pp. 2923-2930

22. Zhang D, Zhao P, Gao J, Zhang T, Gong C. "New method of intelligent data transmission for IoV". Chinese Journal on Internet of Things, 3(2), 2019, pp. 89-99.

23. Zuo X, Wang M, Cui Y. "Low-latency networking: architecture, key scenarios and research prospect". Journal on Communications, 40(8), 2019, pp. 22-35.

24. Ding N, Lin T, Song C, Tan G. "Requirements-driven and multihomed-based multipath TCP congestion control algorithm for vehicular network". Journal on Communications, 2016, 37(7), pp. 96-106.

25. Jiang H, Luo Y, Zhang Q Y, Yin, M., Wu, C “TCP-Gvegas with prediction and adaptation in multi-hop ad hoc networks". Wireless Networks, 23(5), 2017, pp. 1535-1548.

26. Guo Y, Li Z, Yang Z. "Performance Analysis of A-MSDU and UDP in IEEE 802.11 Wireless Linear Multi-hop Network". In: Proceedings of the 2020 IEEE 10th International Conference on Electronics Information and Emergency Communication (ICEIEC), Beijing, China: IEEE, 2020, pp. 22-25.

27. Fu Z, Greenstein B, Meng X, Lu, S. "Design and implementation of a TCP-friendly transport protocol for ad hoc wireless networks". In: Proceedings of the 10th IEEE International Conference on Network Protocols, 2002, Paris, France: IEEE, 2002, pp.216-225. 